Rev. Salud pública. 6 (Sup. 1):40-49, 2004

\title{
Evaluación del Programa de Control de la Malaria en la Amazonía Colombiana
}

\author{
FRANCY Y. PINEDA GRANADOS ${ }^{\mathbf{1}}$, VICTORIA VALERO ${ }^{2}$ y CARLOS A. AGUDELO C. ${ }^{3}$
}

${ }^{1}$ Bacterióloga. M. Sc. Infecciones y Salud en el Trópico. Investigadora Independiente. E-mail:wala109@hotmail.com.

${ }^{2}$ Bacterióloga. M. Sc. Epidemiología. Investigadora independiente. E-mail: mvvalerob@unal.edu.co

${ }^{3}$ Médico. M. Sc. Salud Pública. M. Sc. Ciencias. Instituto de Salud Pública, Facultad de Medicina, Universidad Nacional de Colombia. E-mail: caagudeloc@unal.edu.co

Recibido 18 Febrero 2004/Enviado para Modificación 14 Septiembre 2004/Aprobado 29 Septiembre 2004

\section{RESUMEN}

Objetivo Evaluar el programa de control de la malaria en el Departamento del Amazonas Colombiano, antes y después de la creación del Sistema General de Seguridad Social en Salud-SGSSS.

Métodos El estudio se llevó a cabo en las cabeceras municipales de Leticia y Puerto Nariño, y en los corregimientos de Tarapacá y La Pedrera. Participaron los actores e instituciones relacionadas con el programa de control de la malaria en el Departamento. Se recolectó información primaria y cualitativa, mediante entrevistas semi-estructuradas a los actores, encuestas aplicadas a instituciones y grupos focales seleccionados de la comunidad.

Resultados Antes y después de la creación del SGSSS el programa de control de la malaria en el Amazonas ha tenido grandes dificultades especialmente relacionadas con la accesibilidad geográfica, los desplazamientos de población, la falta de continuidad del programa, el seguimiento y evaluación de las actividades. En la actualidad, los municipios requieren el apoyo administrativo de la Secretaría de Salud Departamental, el recurso humano es insuficiente y de alta rotación, existe poca participación intersectorial y ciudadana, y se destinan pocos recursos a las actividades de promoción y prevención. Existe insuficiente aseguramiento al SGSSS e inapropiado manejo de las aseguradoras, poca capacidad estructural de las instituciones prestadoras de los servicios-IPS y una red diagnóstica de baja cobertura.

Conclusiones Los actores e instituciones que están relacionados con el programa de control de la malaria no están articuladas en un plan específico 
que garanticen el control adecuado de la malaria. El estudio muestra que el SGSSS no ha contribuido de manera decisiva a mejorar la situación de transmisión de la malaria en la Amazonía colombiana.

Palabras Claves: Malaria, prevención y control, ecosistema Amazónico, vectores de enfermedades, grupos focales (fuente: DeCS, BIREME).

\section{ABSTRACT \\ Evaluation of the malaria control program in the Colombian Amazon region}

Objective The purpose of this study was to evaluate the malaria control program in the Amazonas department, Colombia, before and after the creation of the General System for Social Security in Health (SGSSS).

Methods The study was carried out in the municipalities of Leticia an Puerto Nariño, and in the villages of Tarapacá and La Pedrera with the participation of the people and institutions related to malaria control in the department. Primary and qualitative information was collected through semi-structured interviews applied to the people, and through surveys applied to both institutions and selected focal groups from the community.

Results The malaria control program in the department of Amazonas has had great difficulties, both before and after the creation of the SGSSS, particularly related to the geographic accessibility, the displacement of population, the lack of continuity of the program, and the follow-up and evaluation of control activities. Currently, the municipalities require administrative support from the departmental health secretariat, human resources are insufficient and of high rotation, there is little citizen and intersectorial participation and scarce resources are allocated to promotion and prevention activities. Insurance to the SGSSS is insufficient and health insurance companies are inadequately managed, there is little structural capacity of health service providing institutions (IPS) and the diagnostic network has low coverage.

Conclusions The people and institutions involved with malaria control are not articulated in a specific plan which can guarantee the adequate malaria control. The study demonstrates that the SGSSS has not contributed in a decisive manner to improve the malaria transmission situation in the Colombian Amazon region.

Key Words: Malaria, prevention and control, ecosystem, disease vectors, focus groups (source: MeSH,NLM).

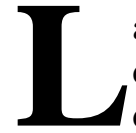

a malaria es un grave problema de salud pública en el ámbito mundial debido a que se presentan anualmente entre 300 y 500 millones de casos clínicos y mueren más de un millón de personas, el $90 \%$ niños menores de cinco años. En las Américas, el 38,4 \% de personas viven 
en áreas con condiciones propicias para la transmisión. El 86 \% de malaria se presenta en países Amazónicos (1).

Cerca del $85 \%$ del territorio colombiano está situado por debajo de 1 500 msnm, presenta condiciones climáticas, geográficas y epidemiológicas aptas para la transmisión de la enfermedad. El tipo de transmisión corresponde a zonas inestables, con patrones endémicos y epidémicos variables y focales en las diferentes regiones (2).

Colombia ha implementado desde 1956 programas de erradicación, financiados y controlados desde el nivel central. En 1969, el programa de control asumió funciones de detección activa-pasiva de casos, diagnóstico, tratamiento y control de vectores. En 1986 se inició el proceso de descentralización, por medio del cual se delegaron y transfirieron progresivamente a los Departamentos y municipios la planeación y ejecución del control de las enfermedades tropicales (3).

La creación del SGSSS en 1993 cambió el esquema centralizado con que funcionaban los programas dirigidos al control de las enfermedades como la malaria. Se estableció el Plan de Atención Básica-PAB, como un medio para coordinar, orientar y ejecutar en los distintos niveles del Estado, el desarrollo de las acciones de promoción, prevención y vigilancia. De otra parte, el Plan Obligatorio de Salud-POS debe garantizar el acceso al diagnóstico y tratamiento de la malaria de la población afiliada al SGSSS.

En el contexto de la descentralización y la reforma del sistema de salud, se hace necesario precisar y evaluar las nuevas problemáticas, los obstáculos y factores favorecedores que inciden en el programa de control, como sistema administrativo, y en las actividades que este debe realizar.

El Departamento del Amazonas representa gran parte de las tradiciones, la historia, los modelos de desarrollo y las problemáticas de salud y enfermedad del Amazonas geográfico. En medio de los procesos de cambio de los sistemas productivos, de las migraciones humanas, las transformaciones sociales y políticas, y la colonización, el Departamento del Amazonas es una unidad ecológica que opera con relativa independencia de otras regiones de Colombia y cuenta con un patrón malárico endémico propio, de bosque húmedo tropical, con una amplia diversidad vectorial, y con sus propios problemas en el desarrollo de las políticas y programas de control de la malaria (4).

\section{MÉTODOS}


Se realizó un estudio evaluativo en el Departamento del Amazonas, en los municipios de Leticia y Puerto Nariño (áreas de mediano y bajo riesgo de transmisión de malaria), y en los Corregimientos de La Pedrera y Tarapacá (áreas del alto riesgo de transmisión). Se utilizaron como parámetros de evaluación categorías definidas previamente tales como las políticas de ETV, la descentralización, el Plan de Desarrollo, el plan local de salud, el $\mathrm{PAB}$, la articulación intersectorial, la participación social, el alcance y cobertura de los programas de ETV, el acceso al diagnóstico y tratamiento de malaria, la vigilancia y los problemas prioritarios.

En la medida que los municipios tienen responsabilidades definidas con respecto al control de la malaria se consideró indispensable incluir a los actores que operan en los mismos. Los actores que participaron en el estudio fueron los siguientes:

Alcalde del municipio de Puerto Nariño, el Ex secretario de Salud departamental del Amazonas, los Secretarios de Salud, del Amazonas y del municipio de Leticia, Administrativos Corregimentales de Tarapacá y la Pedrera; Coordinadoras del Programa de Malaria, de la Unidad de Entomología, del Laboratorio de Salud Pública, de la Oficina de Participación Social, de la Oficina de Seguridad Social, de la Secretaría de salud Departamental del Amazonas; la Coordinadora del PAB municipal de Puerto Nariño, la Coordinadora de ETV y saneamiento ambiental del municipio de Leticia, los técnicos de saneamiento ambiental de La Pedrera y Tarapacá y 3 bacteriólogos que laboraban en el programa de control de malaria de Puerto Nariño, La Pedrera y Tarapacá.

Así mismo, participaron 10 Curacas o representantes indígenas ante el cabildo de las comunidades indígenas del área urbana y rural de Leticia, Tarapacá y Puerto Nariño, 3 Capitanes de las comunidades indígenas de La Pedrera, los Presidentes de la Asociación de Cabildos indígenas del Trapecio Amazónico-ACITAM, de la Asociación de Indígenas de Tarapacá-CIMTAR y de la Asociación PANIT de La Pedrera, y 20 promotores de Salud de las comunidades Indígenas del área rural de Leticia, Puerto Nariño, Tarapacá y La Pedrera.

La información primaria se obtuvo directamente de los actores en los municipios y corregimientos, por medio de entrevistas semi-estructuradas, y de las instituciones por medio de encuestas institucionales. 
Se contó con la participación de funcionarios de una Entidad Promotora de Salud-EPS y de una Administradora del Régimen Subsidiado-ARS, del Hospital San Rafael de Leticia, y los Hospitales Locales de primer nivel de atención de Puerto Nariño, La Pedrera y Tarapacá; también participaron 3 IPS de carácter privado del municipio de Leticia.

Los datos cualitativos se transcribieron literalmente, utilizando las palabras y expresiones de los participantes. Se ubicaron los datos en archivos de categorías y componentes por actores, se sintetizaron, y recontextualizaron. La información primaria y secundaria de tipo cualitativo se interpretó con el método de la teoría fundamentada $(5,6)$, para organizar, sintetizar y dar significado a los datos.

\section{RESULTADOS}

Tendencias de la malaria en el Departamento del Amazonas

Como se observa en la Figura 1, la transmisión de la malaria en el Departamento ha venido incrementándose. En 1960 mostró un Índice Parasitario Anual-IPA de 1,3 por mil habitantes; a mediados de la década de los ochenta aumentó a 4,4 y en 1992 se presentó un incremento hasta 26,2 por mil habitantes; entre 1992 y 1996 el IPA descendió, pero luego se incrementó nuevamente hasta alcanzar un valor de 41,3 por mil en 1999. Hacia el año 2002 el IPA descendió a 11,1 por mil habitantes.

La Figura 2, permite observar la comparación entre el IPA por municipios y corregimientos.

Programa de Enfermedades Transmitidas por Vectores-ETV y Plan de Atención Básica-PAB

El estudio encontró que en los municipios de Leticia y Puerto Nariño se requiere el apoyo técnico y operativo de la Secretaría de Salud Departamental, para la planeación y ejecución del programa de ETV incluido dentro del PAB municipal; así mismo, falta claridad en las competencias que deben asumir. Tanto a nivel Departamental como municipal coinciden en que las causas principales que originan el incumplimiento y la poca cobertura de las actividades planteadas en los Planes Operativos Anuales, son la escasa accesibilidad geográfica y la disponibilidad de recursos financieros. Los recursos insuficientes impiden contar con los medios de transporte que se requieren para responder a la dispersión geográfica de las comunidades, por lo que las acciones no tienen la continuidad ni el seguimiento que se requiere. 
De otra parte, se encontró que los recursos asignados en el PAB departamental y municipal se invierten principalmente en compra de insumos quí-

Figura 1. Tendencias de la malaria en el Departamento del Amazonas. IPA (Indice Parasitario Anual), IFA (Incidencia anual por Falciparum), IVA (Incidencia anual por Vivax). Tasas por mil habitantes $1960-2002$

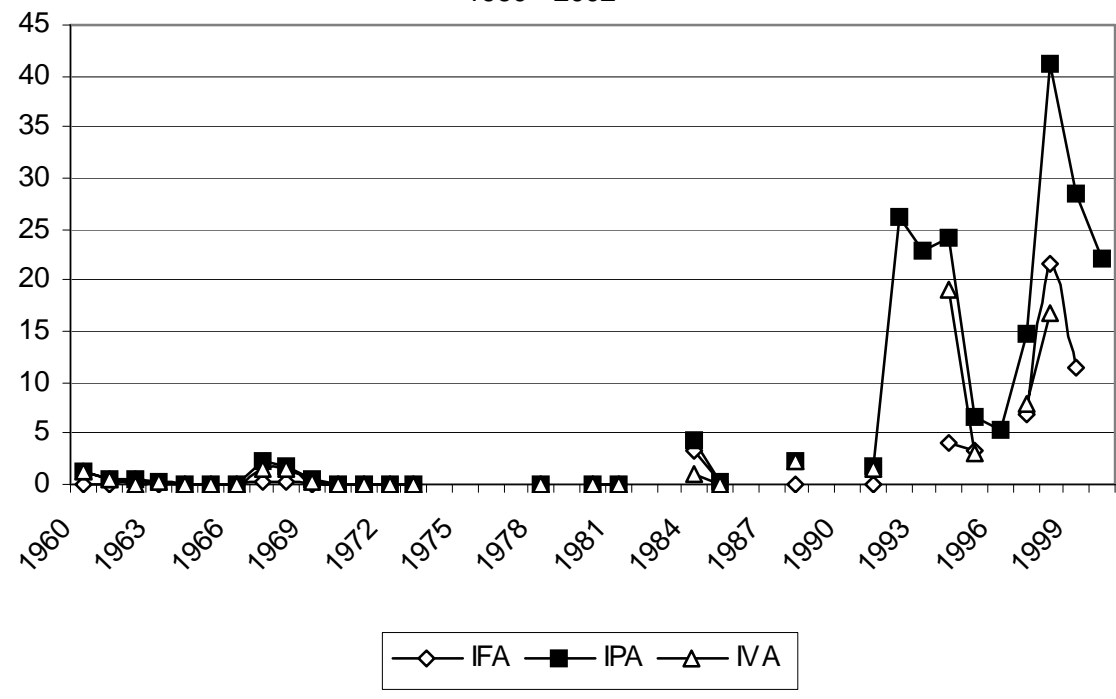

micos, destinando pocos recursos a las actividades de promoción y prevención.

Fuente: Ministerio de la Protección Social. Instituto Nacional de Salud. Programa de control de vectores. Subdirección de epidemiología. 2003.

Figura 2. IPA según municipios y corregimientos. 1995 - 2002

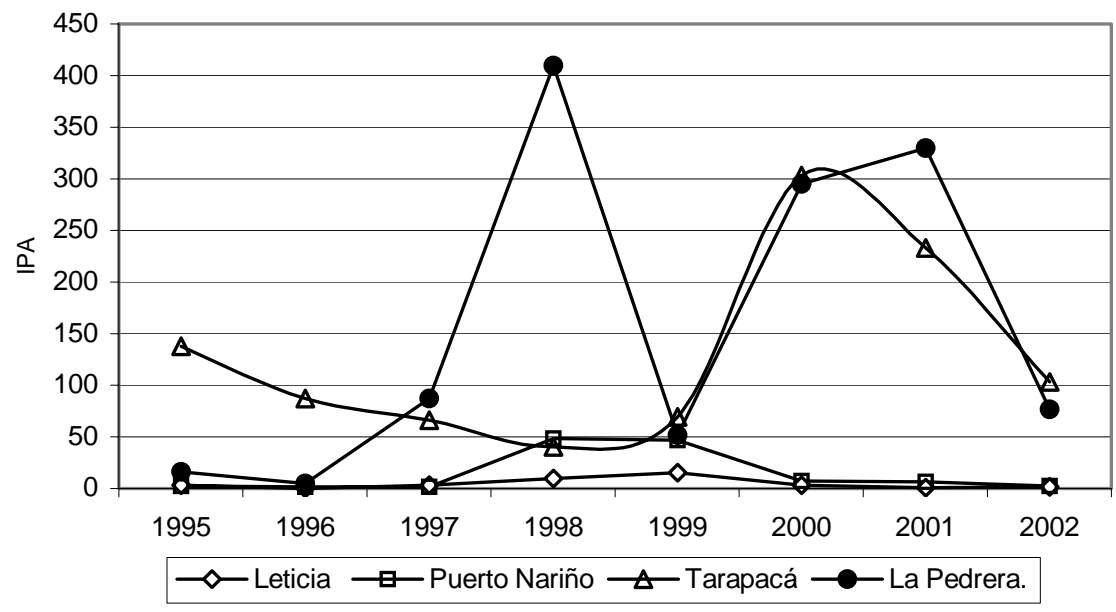




\section{Recurso humano del Programa de Control de ETV}

En el nivel Departamental el programa cuenta con personal capacitado y estable, lo que no ocurre en el nivel municipal, donde el recurso humano presenta una alta rotación y debe capacitarse continuamente. El programa cuenta con promotores de salud y técnicos de saneamiento ambiental, pero no están suficientemente capacitados; en La Pedrera y Tarapacá zonas de alto riesgo para malaria, el personal capacitado que depende de la Secretaría Departamental de Salud, permanece la mayor parte del tiempo en Leticia.

\section{Acción intersectorial para el control de vectores}

El estudio encontró que la participación de otros sectores, diferentes de los directamente responsables del Programa, tanto a nivel Departamental como municipal, no es continua ni suficiente. Así mismo, no existen planes estructurados para la participación ciudadana.

Capacidad y coordinación para garantizar el diagnóstico y tratamiento oportuno de la malaria

Las Instituciones Prestadoras de los Servicios de salud, a cargo del Plan Obligatorio de Salud-POS, no han creado grupos especiales para garantizar la prestación del servicio en zonas de difícil acceso o alejadas de las cabeceras de los corregimientos de La Pedrera y Tarapacá; de otra parte, no disponen de una red de microscopia en estos lugares. A esto debe sumarse que los promotores de salud no disponen de medios de transporte (bote, motor y gasolina), para remitir a los pacientes y la gota gruesa. Esta situación refleja, en gran parte, la falta de coordinación entre Alcaldías, Resguardos Indígena, ARS y el Programa de Control de la Malaria.

\section{Afiliación de la población al SGSSS}

El Departamento del Amazonas presenta un 39 \% de su población afiliada al SGSSS; de esta el $28 \%$ corresponde a los afiliados al régimen subsidiado y el $11 \%$ a los afiliados al régimen contributivo. En el Departamento existe un $61 \%$ de la población no afiliada al SGSSS. El porcentaje de afiliación al régimen contributivo en Leticia corresponde al 27,3 \% del total de población, mientras que en Puerto Nariño es sólo del 0,2 \%; en los corregimientos de la Pedrera y Tarapacá no hay afiliados al régimen contributivo. Los afiliados al régimen subsidiado en el municipio de Leticia corresponden al 88,5 \% de la población, en Puerto Nariño son el 93,2 \% y en los corregimientos de la Pedrera y Tarapacá son 4,7\%. 
Financiamiento del diagnóstico y tratamiento de la malaria

En los corregimientos existe la mayor proporción de personas sin afiliar por lo que continúan recibiendo diagnóstico y tratamiento mediante recursos a la oferta y recursos de las transferencias nacionales.

Especialmente en los corregimientos de La Pedrera y Tarapacá se han presentado inconvenientes para la facturación de la gota gruesa. La población de alto riesgo es atendida por los trabajadores del programa de ETV en el sistema de búsqueda activa, utilizando recursos que ya están financiados en el POS; la población que pertenece a los regímenes contributivo y subsidiado es atendida por los promotores de salud financiados con recursos de la oferta.

\section{DISCUSIÓN Y CONCLUSIONES}

Los resultados del estudio coincidieron con una encuesta transversal realizada en el año 2000 por el Ministerio de Salud y la Organización Panamericana de la Salud, en la cual se encontraron problemas en la implementación del SGSSS y en las actividades de control del Programa de Malaria, tanto en el Plan Obligatorio de Salud como en el Programa de Atención Básica (7)

Las EPS, ARS e IPS no han contribuido a mejorar la situación en cuanto al diagnóstico y tratamiento oportuno de la malaria. De otra parte, las Alcaldías, y las ARS u Organizaciones indígenas, no están articuladas en un plan específico para garantizar el diagnóstico y tratamiento mediante búsqueda activa.

En el Departamento del Amazonas las ARS contratan a la red de IPS de la Secretaría Departamental, que es la única del Departamento, y que no demuestra capacidad estructural para garantizar la cobertura total en diagnóstico. Debe recordarse que sólo el 7,8 \% de las EPS y ARS en el país han generado la estructura necesaria para atender este tipo de problemáticas con puestos propios de diagnóstico.

El programa de ETV termina asumiendo actividades en diagnóstico y tratamiento que deberían asumir las ARS, y esto lleva tales actividades se cubran con recursos provenientes de las transferencias nacionales, lo cual oculta el incumplimiento de las aseguradoras. 
Un problema importante que se evidencia es la poca oferta de ARS o EPS con experiencia en el manejo de comunidades indígenas, mientras que la Iniciativa de Hacer Retroceder el Paludismo-IHRP hace énfasis en la gravedad del problema para los pueblos indígenas.

Las debilidades en el régimen subsidiado, el aseguramiento insuficiente, el inapropiado manejo de las Aseguradoras, la poca capacidad estructural de las IPS y una red diagnóstica de baja cobertura, terminan incidiendo negativamente en el Programa de Control de la Malaria. A lo anterior debe sumarse uno de los aspectos determinantes de la difícil situación actual de las acciones de vigilancia de interés en salud pública, como son las deficiencias técnicas y la insuficiencia e inestabilidad del recurso humano contratado en el nivel local. Igualmente las grandes limitaciones de acceso hacen que operacionalmente el programa de control sea más costoso por los gastos en desplazamiento; esto coincide con el hecho de que en Colombia la mayoría de los casos de malaria provienen de áreas rurales, donde la población es dispersa y en donde factores sociales y geográficos dificultan la operatividad de acciones regulares de control.

En el desarrollo de los elementos planteados en la IHRP, bajo el título de "prevención múltiple y acciones bien coordinadas", las entidades territoriales deben fortalecerse para liderar acciones de control de factores de riesgo ambientales, en la trasmisión de la malaria. Entre los elementos que plantea la IHRP se hace énfasis en la búsqueda de alternativas de control en las que la comunidad sea partícipe de las acciones encaminadas a protegerse de la enfermedad. Adicionalmente, en el Amazonas, por su multiculturalidad, debe tenerse en cuenta la diversidad y particularidad de cada localidad, de tal manera que la participación de la comunidad como estrategia y las acciones de control deben concentrarse en las acciones reguladas a escala local.

Aunque el programa continúa con grandes dificultades, es importante apoyarse en las oportunidades como la creación de puestos diagnósticos en zonas de difícil acceso, capacitación a promotores de salud en microscopia y vigilancia, coordinación intersectorial para financiar y coordinar las actividades en el PAB •

\section{REFERENCIAS}

1. Ministerio de la Protección Social. Instituto Nacional de Salud. Informe Quincenal Epidemiológico Nacional. 2002; 7(19):333. 
2. Ministerio de la Protección Social. Instituto Nacional de Salud. Informe de Situación del Programa Nacional de Vigilancia y Control de la Malaria en Colombia durante el año 2001; Bogotá.

3. Ministerio de Salud. Dirección de Campañas Directas. Informe XV reunión de jefes de zonas de Servicio de Erradicación de Malaria Colombia. Bogotá; 1989.

4. Perez L, Suarez M, Murcia L, de La Hoz F, Olano V, Brochero H, Toro P. La malaria en el Amazonas: conocimientos, prácticas, prevalencia de parasitemia y evaluación entomológica. Biomédica 1999; 19 (2):93-102.

5. Wayne A [Internet]. ¿ Glaser or strauss? grounded theory and adult education. Disponible en: http://www.fundamentada/winniny graduate research paper from the 1996. conference.htm. Consultado Diciembre 2001.

6.Brian D [Internet]. Grounded theory as scientific method. 1996. Disponible en: http://www.Teoría fundamentada/Haig grounded theory as scientific method. Htm. Consultado Enero 2002.

7. Ministerio de Salud. Organización Panamericana de la Salud. Reunión de Impulso a la Iniciativa de Hacer Retroceder el Paludismo (IHRP) en Colombia. Bogotá; 2000. 